\title{
Erratum
}

\section{Influence of a HTR2B Stop Codon on Glucagon Homeostasis and Glucose Excursion in Non-Diabetic Men}

R. Tikkanen, T. Saukkonen, M. Fex, H. Bennet, M.-R. Rautiainen, T. Paunio, M. Koskinen, R. Panarsky, L. Bevilacqua, R. L. Sjöberg, J. Tiihonen, M. Virkkunen

Exp Clin Endocrinol Diabetes 2016;

DOI: $10.1055 / \mathrm{s}-0042-109263$

Article Titel, legend of Table 1, legend of Figure 1 and first six references are corrected. 